\title{
Biometric Iris Recognition of Person from an Image at Long Distance using Chronological Monarch Butterfly Optimization based Deep Belief Network
}

\author{
Swati D. Shirke, C.Rajabhushanam
}

\begin{abstract}
Now days, for the identification of personal information of a person, biometrics is mostly used. Also for the personal identification, the recognition of eye based biometric feature extraction is the most powerful tool. The biometric is an important identity to identify the individual. But in real time it is quite difficult to capture the better quality of iris images. The images obtained are more degraded due to the lack of texture, blur. In this paper, more convenient method is presented for extracting the features of biometric images. The method Iris Recognition at-a Distance (IAAD) is used to extract the iris features of biometric image and to enhance the quality of an image in a biometric system. The Chronological Monarch Butterfly Optimization -based Deep Belief Network (Chronological MBO-based $\mathrm{DBN}$ ) is proposed for iris recognition to get better accuracy. The Monarch Butterfly Optimization algorithm is used to arrange the Chronological assumption of an iris image. Also, the Hough Transform algorithm is used for detection of iris circle and edge. The scaT T loop descriptor and the Local Gradient Pattern (LGP) are used for feature extraction, which is fed to the Chronological MBO-based DBN for iris recognition that enhances the accuracy. The Daugman's rubber sheet model, median filter and trained neural network are used for normalization and segmentation. The UBIRIS.v1 database is used to take an iris recognition images and MATLAB is used for programming of for reading the iris images and for performing the Hough transform operations. The iris recognition at a distance 4 to 8 meter is done with the help of simulation result. The performance is analyzed based on the metrics, like False Acceptance Rate (FAR), accuracy, and False Rejection Rate (FRR) with the value of $0.4847 \%, 96.078 \%$, and $0.4745 \%$.

Index Terms: Deep Belief Network, Matlab, Iris recognition, Hough Transform, ScatT-Loop, LGP, Feature Extraction, Dougman's Rubber Sheet Model, etc.
\end{abstract}

\section{INTRODUCTION}

In most of the practical based applications like as congregation entrance, airport boarding, custom clearance and so on requires high security. For this high security purpose most of the companies uses iris recognition system.

The government of India uses this system to identify the citizen in many applications like as Aadhar project, in rashan

Revised Manuscript Received on July 22, 2019.

Ms. Swati D. Shirke, Ph.D.Scholor, Bharath Institute of Higher Education and Research Chennai, Tamil Nadu, India .Email:shirke.swati14@gmail.com

Dr.C.Rajabhushanam, Professor, Computer Science \& Engineering, Bharath Institute of Higher Education and Research, Chennai, Tamil Nadu, India. rajabhushanamc.cse@bharathuniv.ac.in shops, while filling different government exam forms, registration department, etc. But most of these face one problem that, iris at a distance and getting an iris image, also the motion of camera and person. Therefore, the iris recognition requires the intelligent system with high reliability and less coefficient errors [1]. There are two classifications of biometric system namely unimodal and multimodal biometric systems. In unimodal biometric system the identity of the person is based on the single information source, such as left iris, right iris and face etc. [2]. The multimodal biometric system uses the classifier to match the specific person [2]. Iris recognition system uses the following three processing components, namely acquisition, pre-processing, feature extraction [3]. Figure 1 shows the eye sample introduction. Iris uses the gabor filters to extract the global and local facts of the image as the feature vector.

The main objective of this article is to design a method using the machine learning approach. Initially, the iris image is fed into the pre-processing step. In this step the Hough Transform (HT) is used to extract the region of iris and the localization of pupil. For iris image normalization and automatic segmentation the Daugman's Rubber Sheet Model is designed.

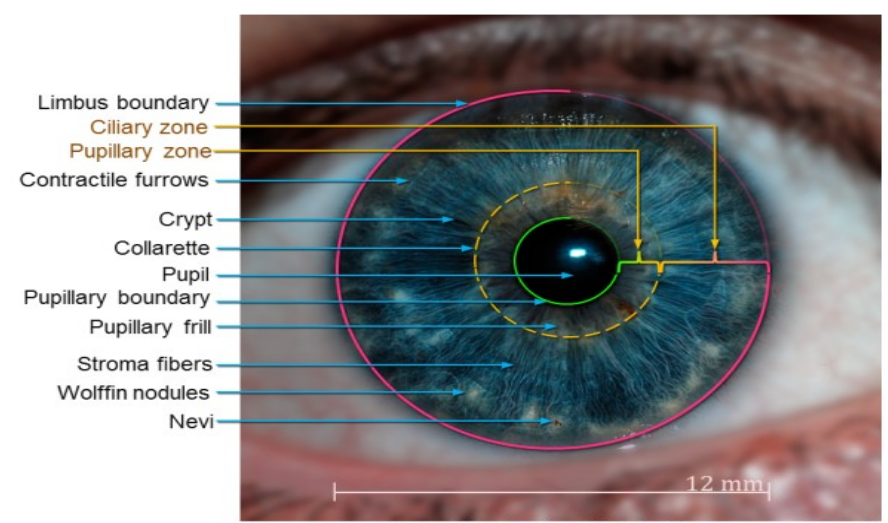

Fig.1 Eye Sample Introduction

The ScatT-Loop descriptor and LGP are used to extract the different features of iris image, where the ScatT-Loop is developed using the Scattering transform (ST), Tetrolet transform (TT),

and Loop descriptor. Once the features are extracted, the recognition is performed using

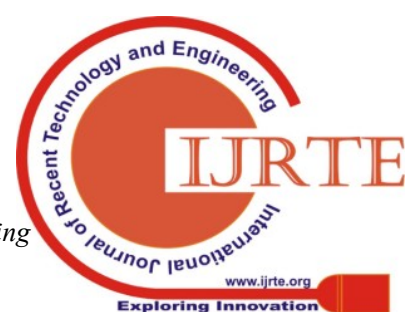


the DBN based on the proposed Chronological MBO algorithm.

The organization of different sections of the paper is given below. For designing the system the various methods used are presents in the section II. Section III represents the block diagram of proposed system. The experimental results are presents in the section IV. Finally the conclusion of the proposed system is presents in the section $\mathrm{V}$.

\section{II.METHODS USED IN PROPOSED SYSTEM}

The iris recognition plays an important role to identify the person based on the features of the iris texture of a person. In this section the different methods used in this iris recognition system are presents.

\section{A. Preprocessing}

The preprocessing stage is used to enhance the different parameters an iris image such as contrast, intensity, signal to noise ratio, etc. Also it is used to increase the quality of an image due to undesired distortions. This step enhances the features of iris image which are required for further processing. It only enhances the quality of image; it does not increases the information content of an image. The intensity range of an iris image is normalized to [0 1]. This range of intensity values shows the maximum intensity value by dividing all intensity values. The diffusion filter process is explained in [11] is used to increase the intensity and contrast of an iris image. The signal to noise ratio of iris image is improved with the help of anisotropic diffusion filter. The tool which is used for this purpose is MatLab realize the many brightness transformations. To find the rank-order information and spatial information of an iris image a weighted median (WM) filter is used and this is one of the type of median filter. The noises shot and impulse noise are rejected by the median filter.

\section{B. The Hough Transform}

To find the edges and lines of an image, the Hough transform is used.The Hough transform finds different shapes like as circles or ellipses of an image. It is also used to improve the quality of edges presents in the image.
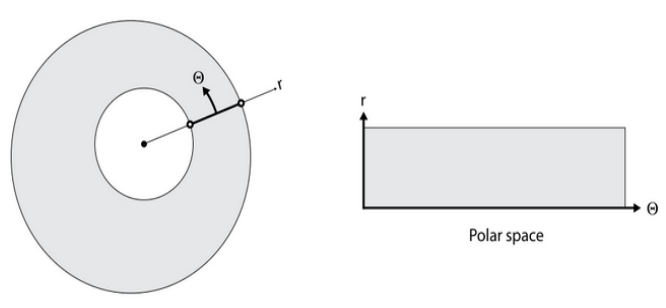

Fig.2. Daugman's rubber sheet model

In this dissertation the Hough transform is used to the iris region from the pre-processed image in order to perform the feature extraction effectively. The line an iris image can be detected by the formula

$$
r=x \cos \theta+y \sin \theta
$$

where, $r$ is quantized distance and $\theta$ is quantized angle. The $r$ and $\theta$ are considering quantized values in the pair $(r, \theta)$.
The boundary of the inner pupil and outer pupil of an iris image can be detected by using the equation,

$$
\left(m-m_{0}\right)^{2}+\left(n-n_{0}\right)^{2}=r^{2}
$$

Where, $\left(\mathrm{m}_{0}, \mathrm{n}_{0}\right)$ denotes the coordinates of a circle with radius r. The results obtained from Hough transform shows the boundary of pupil, eyelid extraction of iris image and the center of pupil.

\section{C.The Normalization and Segmentation}

The normalization and segmentation is the first step of this iris recognition system. The main aim of this method is to detect the two iris borderline, layout, centers, eyelids, eyelashes, radii, etc. This method is also used for to find the location of lower as well as upper eyelid and to separate the eyelashes. The feature encoding, acquisition and image segmentation of an iris image can takes place by using the Daugman's Rubber Sheet Model [12]. Segmentation is performed to retrieve the interested region and the normalization is carried out to minimize the noise in iris to improve the efficiency of recognition. This method uses the Canny edge detection techniques to find the Hough circles on iris images. Figure 2 shows the Dougman's Rubber Sheet Model. After the segmentation, the processed iris image is applied for normalization. In this the segmented iris image is normalized into the block with equal in size respect to the block width $x$ and angular displacement $\theta$. The Daugman's rubber sheet is an linear model that is assigned to the iris of the individual pixel based on the dilation, size and the real coordinates $(\mathrm{m}$, $\theta$ ), where $m$ is the unit interval and $\theta$ ranges from 0 and $2 \pi$. The iris image is remapped into the polar coordinate $(\mathrm{m}, \theta)$ system from cartesian coordinated $(\mathrm{p}, \mathrm{q})$ system. Therefore the normalized polar coordinates are $(\mathrm{m}, \theta)$ and the normal coordinates are $(\mathrm{r}, \mathrm{s})$.

Let the iris coordinates and iris boundaries of the pupil are represented as $\left(\mathrm{r}_{\mathrm{b}}, \mathrm{s}_{\mathrm{b}}\right)$ and $\left(\mathrm{r}_{\mathrm{e}}, \mathrm{s}_{\mathrm{e}}\right)$ along $\theta$ direction. The coefficient of an iris image will not be shifted even if the signal is distorted due to the camera and persons position.

\section{D.ScatT-loop for Feature Extraction \& Feature Matching}

The classified new vessels image of an iris image can be obtained in the feature extraction step. The normalized iris image is subjected to perform the feature extraction by using ScatT-loop descriptor. The ScatT-loop generates the texture features for accurate iris recognition to uniquely identify the individuals. In this, the image region contains many vessel segments that are closely spaced with multiple orientations and have a twisted in nature. From a binary vessel maps, the new vessel segments are generated for the measurement of feature characteristics. Also, a sub window of size $4 \times 4$ is created to find out the local features iris image. This sub window examines the iris image and window the number of vessel pixels and pixel passion can be calculated for every sub window.

The Kirsch mask is designed for the future extraction. The loop value for the corresponding pixel is represented as, 


$$
\operatorname{LOOP}\left(P^{*}, Q^{*}\right)=\sum_{i=0}^{7} h\left(R^{k}-R^{*}\right) \cdot 2^{\nu}
$$

Where,

$$
h(d)=\left\{\begin{array}{lll}
1 & ; & \text { if } d \geq 0 \\
0 & ; & \text { Otherwise }
\end{array}\right.
$$

$\left(P^{*}, Q^{*}\right)$ is the centre of the intensity of iris image. $\mathrm{R}_{\mathrm{k}}$ is the neighborhood pixel intensity, $\mathrm{R}$ is the original image pixel intensity, and $k$ takes the value ranges from 0 to 7 .

\section{E. Extraction of features using Local Gradient Pattern (LGP)}

The LGP generates the constant patterns of face representation of person, which is irrespective to the intensity variation with the edges. The intensity a value of a pixel is determined by gradient pixel values which is operated by LGP operator. The minimum value of the gradient among the eight neighboring pixels is considered as the threshold value. When the gradient value of the neighboring pixel is higher against the threshold, then the value assigned to the pixel is ' 1 ' otherwise the value is ' 0 '. The LGP can be represented as the formula

$$
\operatorname{LGP}_{b, 0}\left(x_{a}, \bar{x}_{a}\right)=\sum_{i=0}^{b-1} p\left(y_{i}-\bar{y}\right) 2^{n}
$$

where,

$$
p(r)= \begin{cases}0, & \text { if } r<0 \\ 1, & \text { otherwise. }\end{cases}
$$

0 is the centre of a circle, $b$ are the sample points on the circle, LGP uses the bilinear interpolation to compute the neighborhood pixel values for 0 and $b$. It uses $(2 \times m+1)$ and $(2 \times m+1)$ kernel to summarize the structure of the iris. Gradient value between the neighboring pixel $n_{i}$ and the center pixel $n_{a}$ is denoted as, $y_{c}=\left|n_{i}-n_{a}\right|$ and define the average gradient value of $b$ as,

$$
\bar{y}=\frac{1}{b} \sum_{i=0}^{b-1} y_{i}
$$

The feature vector is represented as,

$$
\gamma=\left\{\gamma_{1}, \gamma_{2}, \ldots . . \gamma_{w}\right\} ; \text { for }(1<\tau<w)
$$

where, dimension of features computed from the input image $X_{i}$ is represented as [ $1 \mathrm{x} \mathrm{w}$ ] and $\mathrm{w}$ is the total feature dimension.

\section{F. Principal Component Analysis (PCA)}

Principal Component Analysis (PCA) is a method which is used to decrease the dimensionality of an image. This method is also used for multivariate analysis of an image. PCA is a compression technique which compresses the high dimensional vectors of an image into the low dimensional vectors and compute the parameters from the data directly. To reduce the dimensionality of an image, PCA extracts less number of components. The principal components of an image can be extracts by using covariance matrix or multivariate set. The compression and decompression operations of an image ca perform by using matrix multiplication. The PCA model is represented as,

$a_{o c 1}=E_{o c w} c_{w c 1}$

Where, a is an zero dimensional vector with the projection $\mathrm{c}$, and $\mathrm{w}$ is the feature vector dimension as $(0<\mathrm{w})$. The covariance matrix a is denoted as,

$A=\frac{1}{\rho-1} \sum_{\tau=1}^{\rho}(c-\kappa)(c-\kappa)^{G}$

where, $K$ denotes the mean vector of $C$. The Eigen vectors $\beta_{\mathrm{T}}$ is expressed as,

$\left(E-\phi_{\tau} \vartheta\right) \beta_{\tau}=0 \quad ; \quad \tau=1,2, \ldots \ldots . w$

where, $\varnothing_{\mathrm{T}}$ denotes the Eigen vectors of $E$. The projection matrix is calculated as,

$D=J^{G}$

where, $J$ has 0 Eigen vectors and $\mathrm{D}$ is the $o c w$ matrix.

The dimensionally reduced feature vector is represented as,

$\gamma=\left\{\gamma_{1}, \gamma_{2}, \ldots ., \gamma_{\tau} \ldots . \gamma_{o}\right\} ;$ for $(1<\tau<o)$

where, 0 is the dimensionally reduced features with $0<w$.

\section{G. Chronological MBO-based DBN}

The DBN classifier using the Chronological MBO algorithm is used for the identification of a person. The non-linear complex relation presents in the real life are removed by using DBN classifier and the chronological MBO algorithm trains the DBN classifier. The iris recognition is performed using the chronological MBO-based DBN, which is the integration of the chronological concept with the MBO algorithm to train DBN that depends on the migration features of the monarch butterfly. The standard MBO incorporates the fine tuning of parameters and the complex free computation to enhance the performance of the proposed chronological MBO-based DBN, and the high dimensional issues are effectively dealt using MBO. Figure 3 shows the Architecture of DBN classifier.

However, the searching speed and the convergence speed are enhanced by integrating the chronological concept, which defines the solutions (biases and weights) from the preceding iterations to revise the new biases and weights.

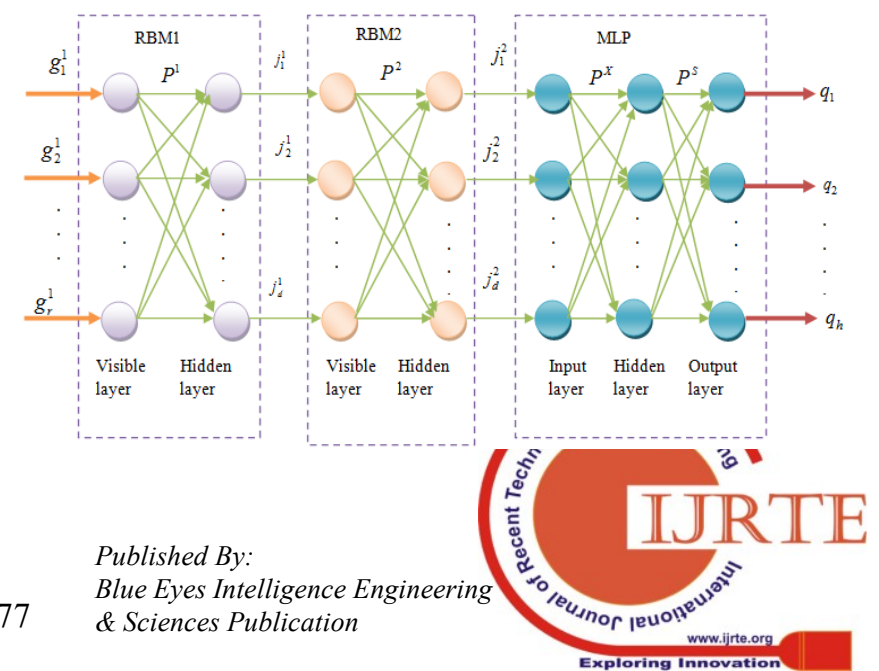


Fig. 3 Architecture of DBN classifier

\section{H. Performance metrics}

The metrics used to evaluate the methods, are FRR, FAR, and accuracy are explained below.

1) Accuracy: The accuracy measures the accurateness of iris recognition based on iris modality and is represented as,

Accuracy $=\frac{T c+T u}{T c+S c+T u+S u}$

where, $T u$ denotes the true positives, and $T c$ is the true negatives. $S u$ denotes the false positives and $S c$ is the false negatives.

2) Sensitivity: Sensitivity is otherwise called True positive Rate (TPR), which is the measure of positive-ness identified correctly, and is calculated using the below equation.

Sensitivity $=\frac{T u}{S c+T u}$

3) False Rejection Ratio (FRR): FRR is the ratio of false rejection to the genuine attempts, and is expressed as,

$F R R=\frac{S c}{T u+S c}=1-T P R$

4) Specificity: Specificity or True Negative Rate (TNR) is the measure of false negatives, which are correctly located. Specificity is expressed as,

Specificity $=\frac{T c}{T c+S u}$

5) False Acceptance Rate (FAR): FAR is the ratio of false attempt to imposter attempts, and is represented as,

$F A R=\frac{S u}{S u+T c}=1-T N R$

6) Receiver Operating Characteristics (ROC): ROC refers to the relationship between TNR and TPR, which is used to compute the performance of the system.

\section{BLOCK DiAgRAM OF PROPOSED SYSTEM}

Here in this unit block diagram of suggested structure is given. The implementation of this work is given in this Step and the details of subparts and algorithms.

\section{A.Test Images}

Here there are 20 peoples dataset is to be considered for the experiment. The database used for this purpose is CASIA.V4 [21][22]. The complete database of this section was looked for Iris images.

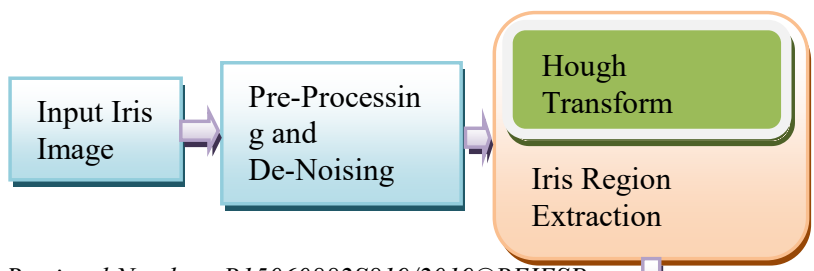

Fig.4 Suggest Method For Iris Recognition.

\section{B.Pre-processing and De-noising}

In pre-processing, the iris image profile is enhanced by calculating signal-to-noise fraction. Iris Recognition at-a Distance (IAAD) is used to enhance the contrast of the image. Median based filters are used to remove the sound presents in the degraded iris images.

\section{C.Hough Transform}

The Hough transform is used to extracts the different curves or shapes of an iris image.

\section{D.Segmentation and Normalization}

In this segmentation step, the iris image is split into dissimilar borders. The division of the regions can take place by considering similar properties 
of an iris image. Some of the similar properties are color, brightness, contrast, texture, gray level, etc. The segmented iris image is prepared by using a normalization algorithm.

The segmented iris image is used for the future extraction process. Due to the varying position of a person and the camera, the iris image is highly affected by distortion [20]. Therefore normalization is used to compensate for this problem. The Daugman's Rubber Sheet Model is use for this purpose.

\section{E. Feature Extraction}

The ScatT-Loop generator is use to extract the different features of an iris image. The ScatT-Loop generates the texture features for accurate iris recognition to uniquely identify the individuals. Operator in LGP utilizes the gradient pixel values and is determined as the intensity value. Different transforms presents in this step are used to calculate the texture features for the iris image. PCA is an optimal scheme to compress the high dimensional vectors into the low dimensional vectors and compute the parameters from the data directly.

\section{F. Deep Belief Network (DBN)}

The dimensionally reduced features obtained using PCA is fed as input to DBN to recognize the individuals. The effective recognition is obtained by the optimal tuning of the DBN classifier using the Chronological MBO algorithm. By using Chronological $\mathrm{MBO}$ algorithm we can forecast or classify accurate Iris image.

\section{EXPERIMENTAL RESULTS}

Here observational outcomes are defined in this unit of suggested system. The step by step execution of the proposed system is presented. Firstly we take input test iris image. In this paper, Around 22,035 iris images from 700 individuals dataset is to be considered for the experiment. The database used for this purpose is UBIRIS.v1 [26]. Figure 3 shows the key in iris recognition image having the dimension $512 \times 512$. This image can be selected by using the GUI in MatLab. This Input Test Image Of The Iris Is Applied For The Pre-Processing. In This Stage, The Different Noise Presents In An Iris Image Are Removed And Increase The Quality Of An Image.

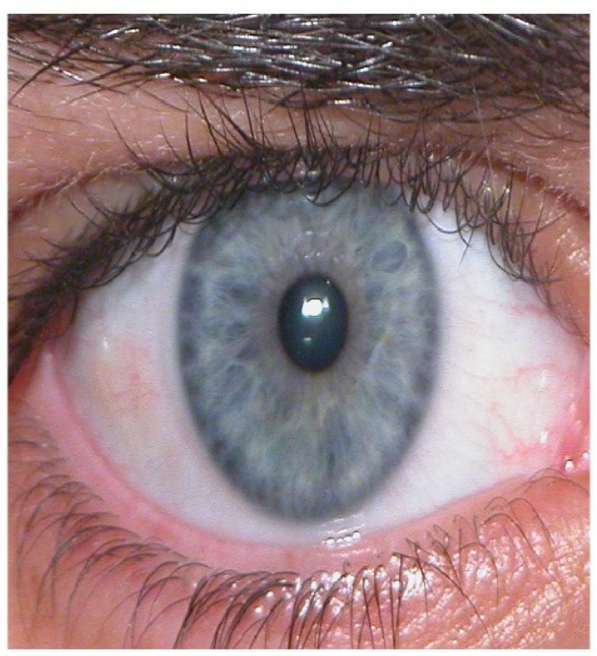

Fig.5 Input Iris Image.

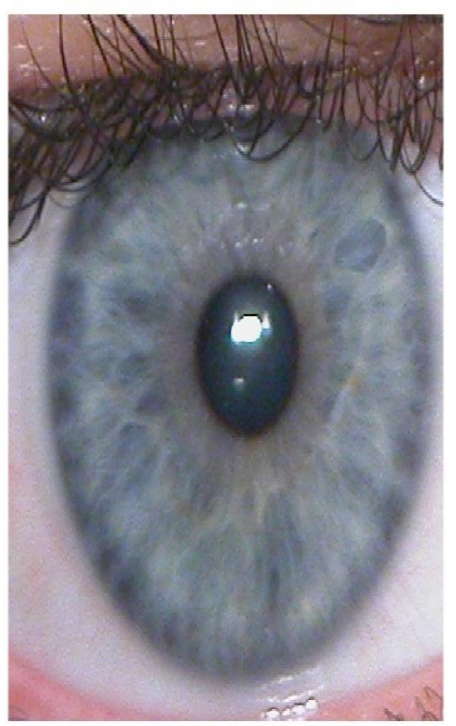

Fig. 6 Processed Iris image.

Figure 5 shows the input image. Figure 6 shows the output of preprocessing the image, which enhances the iris part of the eye (black portion). To eliminate the sound from iris images median filter is used. Figure 7 represents colors to gray as well as resounding image. Color image is the real image, besides of real image is gray image, salt also pepper sound is at the lower level of the original image, also the last one is the Median Filter image. In the Segmentation and Normalization process detection of Pupil and Iris boundary of the original image is takes place. 
Biometric Iris Recognition of Person from an Image at Long Distance using Chronological Monarch Butterfly Optimization based Deep Belief Network

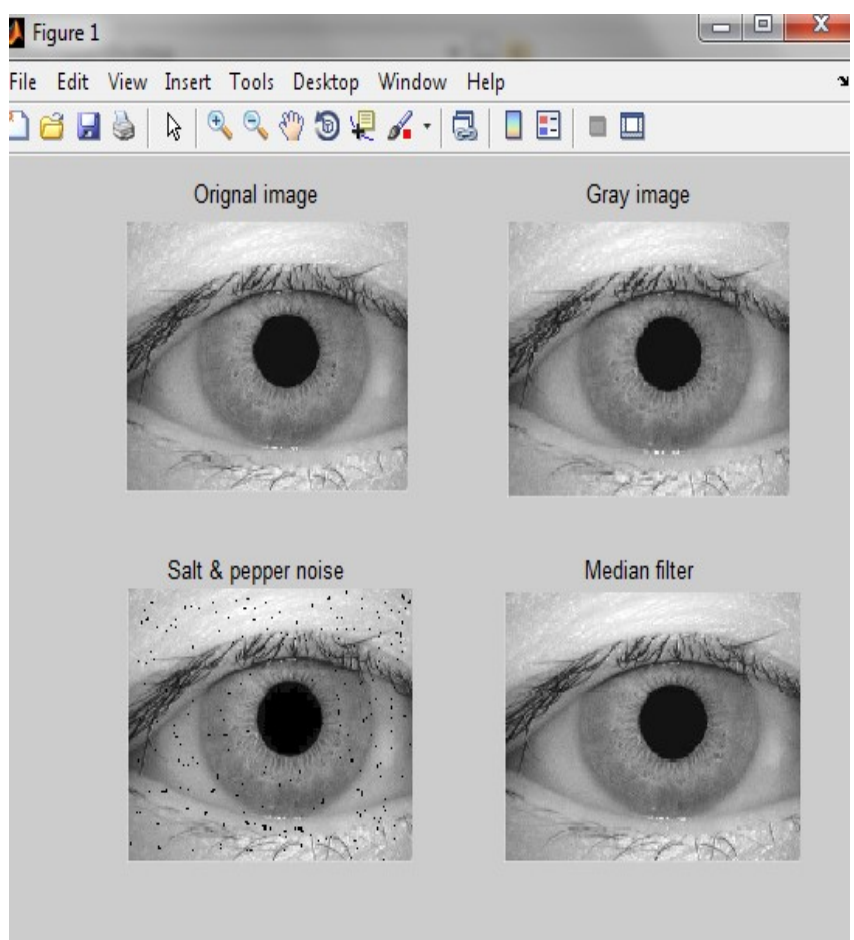

Fig.7 operations on Iris image.

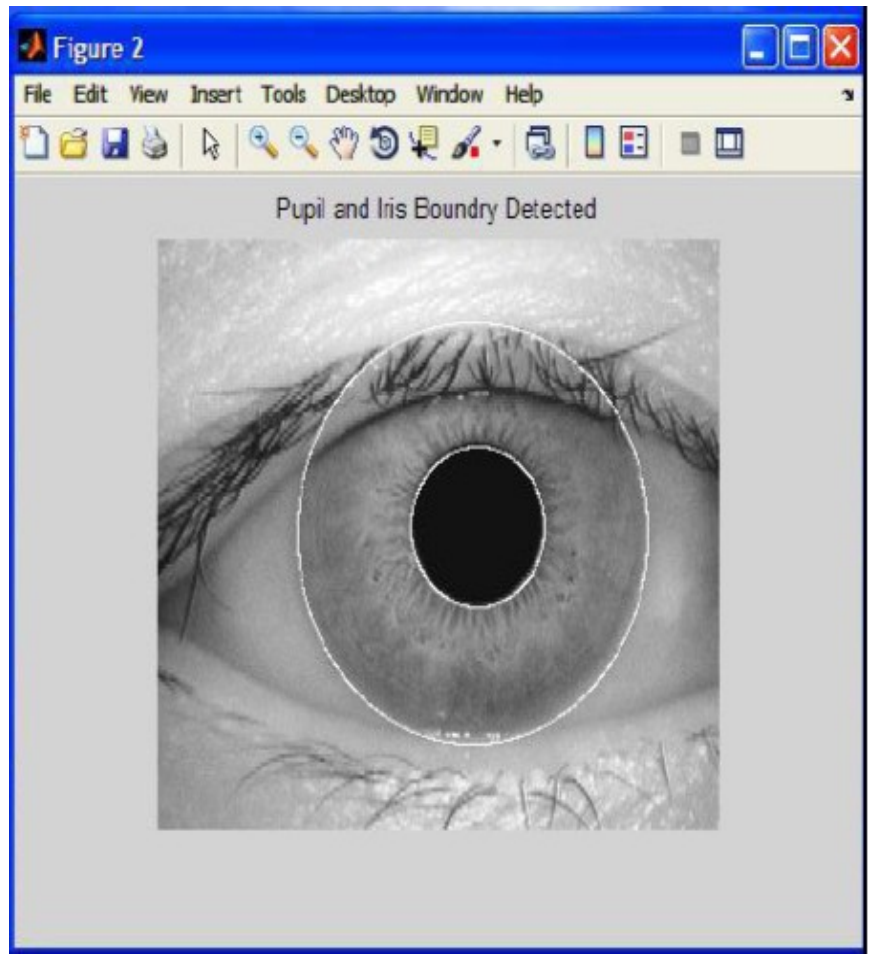

Fig.8 Boundary detection of an Iris Image.

The detection is done with the help of Hough circles. Figure 8 shows the detected boundaries of an iris image. The white border presents in this image is the corresponding boundaries of the iris image. After boundary detection, the edge of an iris image is detected by using Normalization and Segmentation process. Figure 9 shows the corresponding edges of the pupil of an iris image. For finding edge of canny edge detection algorithms are used Figure 10 shows the eyelid of the bottom and top segmentation of an Iris profile.

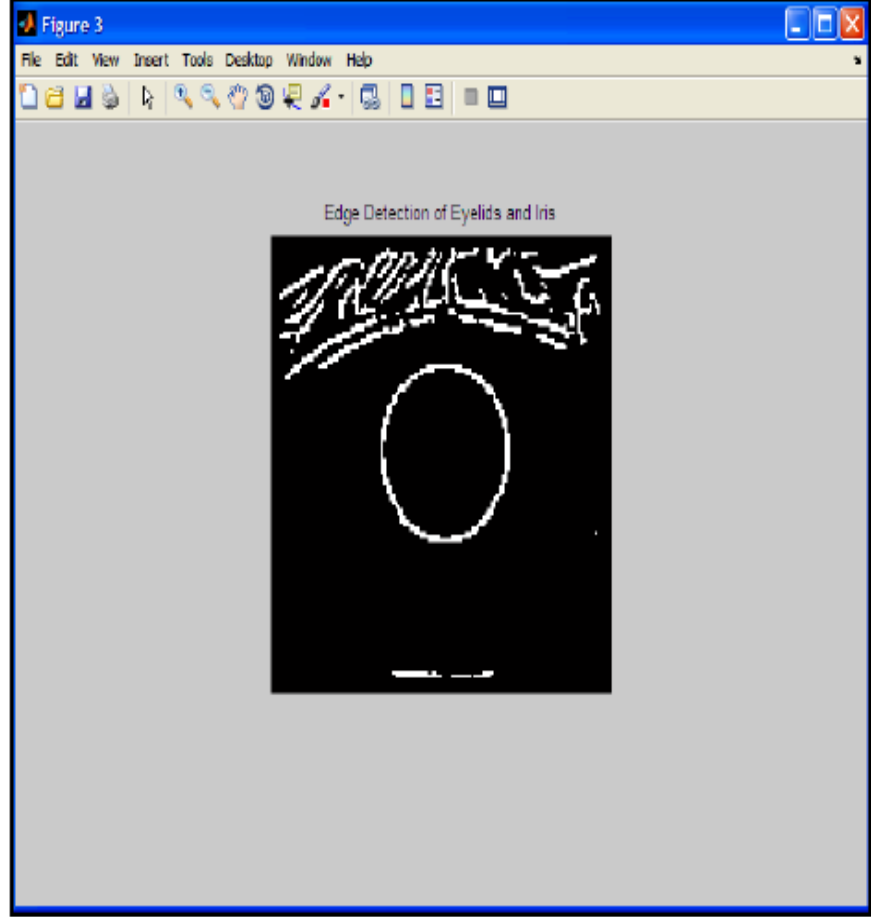

Fig, 9 Edge detection of an Iris Image.

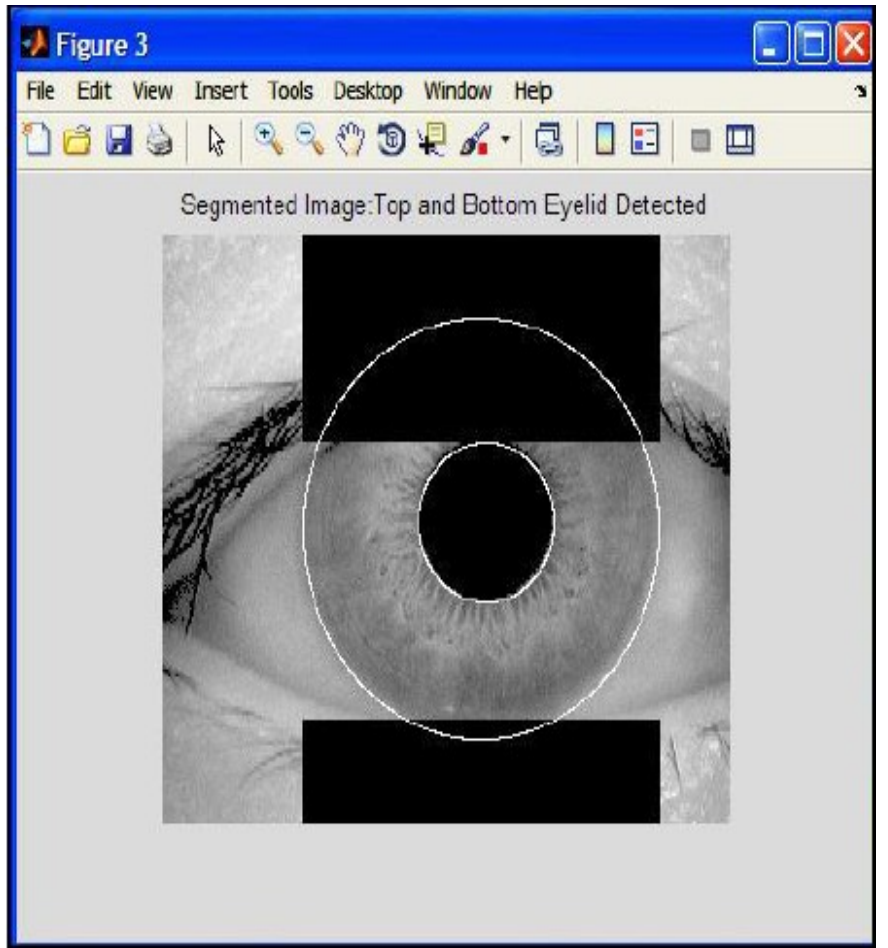

Fig.10 Eyelid of the bottom and top segmentation of an Iris Image.

Figure 9 shows the normalized output image after detection of Iris boundary and Pupil along with bottom and top eyelid recognition and exclusion for Iris Image Normalization and Segmentation. The division of the regions can take place by considering similar properties of an iris image. Some of the similar properties are color, brightness, contrast, texture, gray level, etc. The segmentation identifies the region of interest, finds the location of the tumor, lesion and other abnormalities.

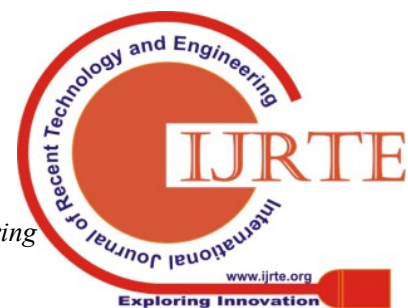




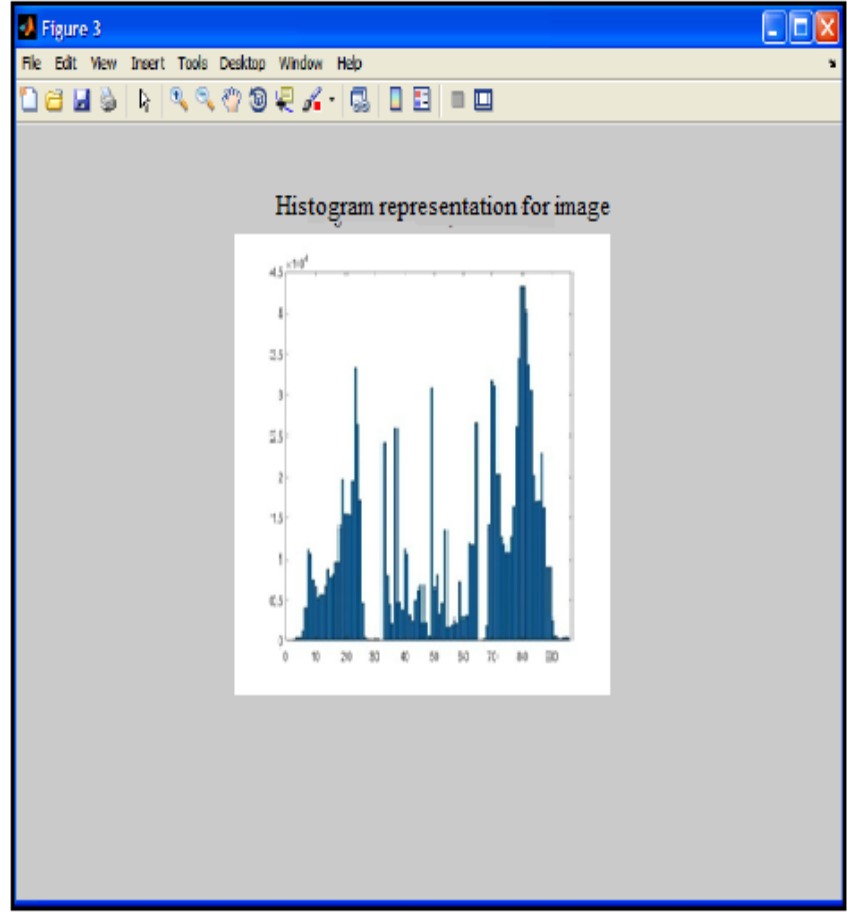

Fig.11 Histogram representation for image.
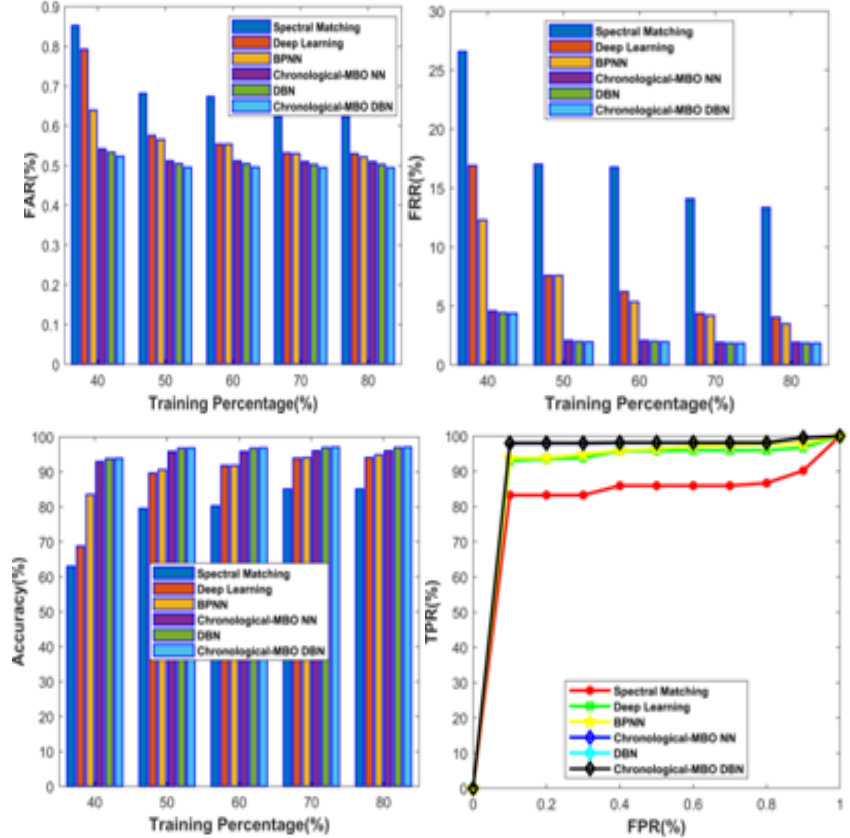

Fig.12 Comparative analysis based on k-fold using image , a) FRR, b) FAR, c) Accuracy and d) ROC analysis.
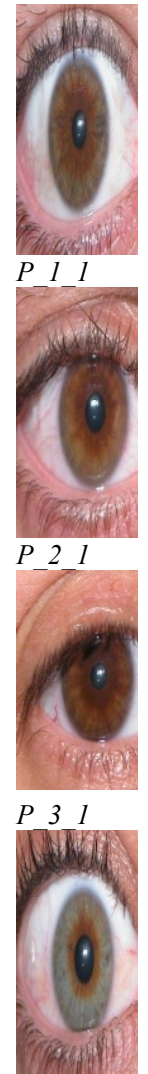

$P \_4 \_1$
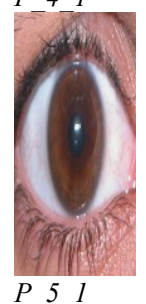

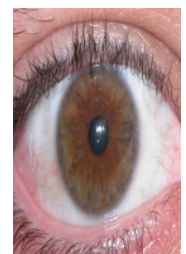

$P \_1 \_2$

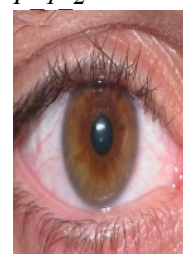

$P 22$

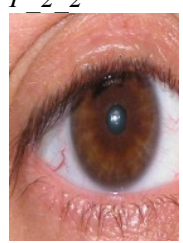

$P 32$

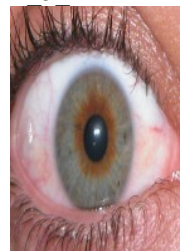

$P \_4 \_2$

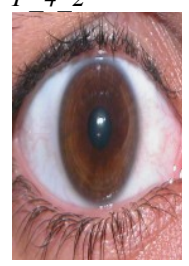

$P 52$

Fig.13. UBIRIS.v1 Database

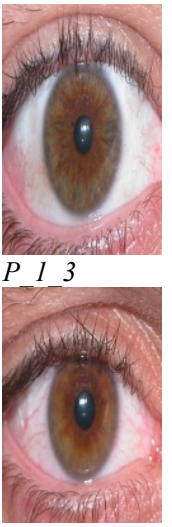

$P 23$

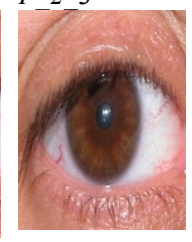

P_4 3

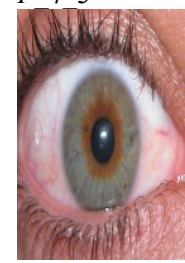

$P 43$

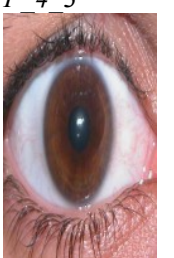

$P 53$

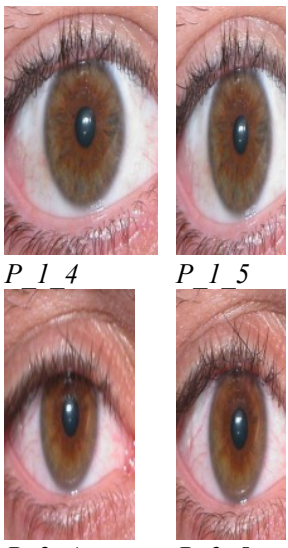

$P \_24$

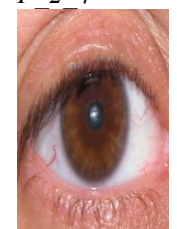

$P \quad 3 \quad 4$

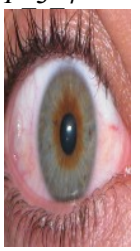

$P 44$

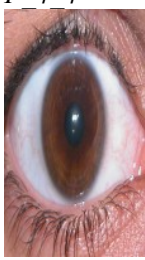

$P 54$

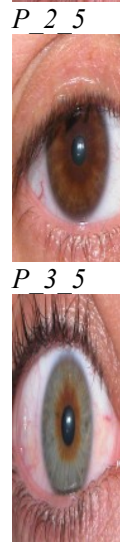

$P 45$

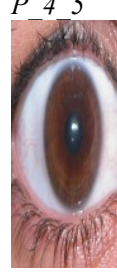

P_5_5

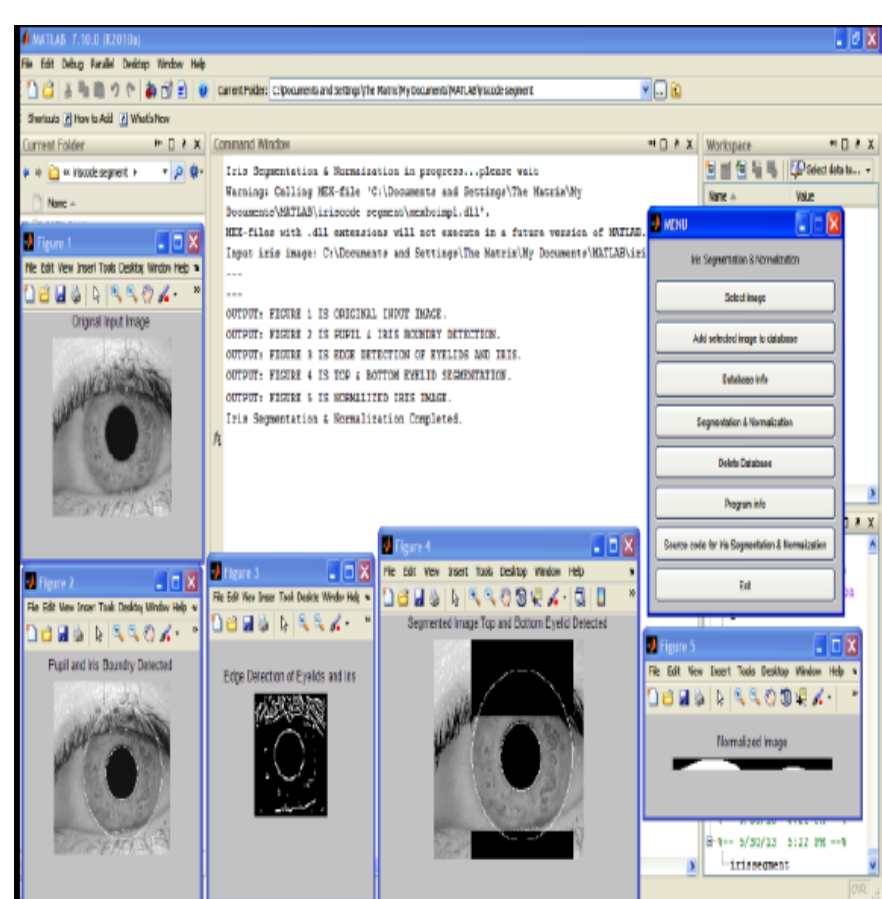

Fig.14 The detailed output of the proposed system.

Blue Eyes Intelligence Engineering 
Figure 11 shows histogram representation for image. Figure 12 depicts the comparative analysis for the metrics, a) shows FAR, b) shows accuracy, c) FRR and d) ROC analysis with respect to the k-fold validation. Figure 14 shows the dataset of different iris images. Figure 14 shows the detailed output of the proposed system.

\section{TABLE I. TABLE STYLES}

\begin{tabular}{|l|l|l|l|l|l|l|}
\hline \multirow{2}{*}{ Methods } & \multicolumn{2}{|l|}{ FRR (\%) } & \multicolumn{2}{l|}{ FAR (\%) } & \multicolumn{2}{l|}{$\begin{array}{l}\text { Accuracy } \\
(\%)\end{array}$} \\
\cline { 2 - 7 } & $\begin{array}{l}\boldsymbol{k} \text {-fol } \\
\boldsymbol{d}\end{array}$ & Training & $\begin{array}{l}\boldsymbol{k} \text {-fol } \\
\boldsymbol{d}\end{array}$ & Training & $\begin{array}{l}\boldsymbol{k} \text {-fol } \\
\boldsymbol{d}\end{array}$ & Training \\
\hline $\begin{array}{l}\text { Spectral } \\
\text { Matching }\end{array}$ & 1.30 & 10.912 & 0.52 & 0.5964 & 85.0 & 63.002 \\
\hline $\begin{array}{l}\text { Deep } \\
\text { learning }\end{array}$ & 1.03 & 2.9 & 0.51 & 0.5261 & 86.3 & 68.764 \\
\hline BPNN & 0.85 & 0.786 & 0.51 & 0.5040 & 91.5 & 83.554 \\
\hline $\begin{array}{l}\text { Chronolo } \\
\text { gical-MB } \\
\text { O-NN }\end{array}$ & 0.5 & 0.786 & 0.5 & 0.5040 & 95.0 & 92.942 \\
\hline DBN & 0.47 & 0.745 & 0.49 & 0.4972 & 95.9 & 93.8 \\
\hline $\begin{array}{l}\text { Chronolo } \\
\text { gical-MB } \\
\text { O based } \\
\text { DBN }\end{array}$ & 0.47 & 0.4745 & 0.47 & 0.4889 & 96.0 & 93.886 \\
\hline
\end{tabular}

Table 1 demonstrates the comparative discussion of the existing and the proposed Chronological MBO-based DBN method. Based on k-fold validation, the FRR rate obtained by the methods, spectral matching, deep learning, BPNN, Chronological-MBO-NN, DBN, and the proposed Chronological MBO based DBN is $1.3055 \%, 1.0372 \%$, $0.8577 \%, 0.5 \%, 0.4798 \%$, and $0.4745 \%$, respectively.

\section{CONCLUSION}

In this paper an effective iris recognition system for person identification is present. The false responses are reduces with the help of binary iris segmentation and in image segmentation process, the unnecessary background images are removed. A method for IAAD is introduced for iris recognition using Chronological MBO-based DBN is proposed in this research work. The optimal weights are determined to train the chronological MBO algorithm using DBN. For the segmentation Hough transform is used. It finds the linear, circular and parabolic segments if an iris image. The extraction of fixed number of features are done with the help of normalization. For testing purpose of this iris recognition system, UBIRIS.v1 database is used. For iris segmentation, normalization, feature encoding and feature matching a Daugman's rubber sheet model is used. The iris recognition based on the features of the biometric is performed using the proposed Chronological MBO algorithm, which is trained using the deep learning approach termed as DBN. The false responses to bright lesions and other retinal feature of eye are reduces by this system. MatLab digital image processing tool is used for this purpose. The human machine interface, image processing and image acquisition problems of an iris image are solved by this proposed system. The experimental results show accurate and successful reorganization the iris upto 4 to 8 meter long. The proposed Chronological based DBN approach attained minimal FRR of $0.4745 \%$, minimal FAR of $0.4847 \%$, and maximal accuracy of $96.078 \%$. The future extension of this work can be made by using any optimization algorithm to enhance the performance of iris recognition.

\section{ACKNOWLEDGMENT}

The authors want to thank the Bharath Institute of Higher Education and Research, Chennai, Tamil Nadu, India.

.Also thanks to STE's NBN Sinhgad School of engineering, Ambegaon (Bk), Pune.

\section{REFERENCES}

[1] Ahmadi N. and Akbarizadeh G., "Hybrid robust iris recognition approach using iris image pre-processing, two-dimensional gabor features and multi-layer perceptron neural network/PSO”, IET Biometrics, vol. 7, no. 2, pp.153-162, 2017.

[2] Alaa S. Al Waisy, Rami Qahwaji, Stanley Ipson, Shumoos Al Fahdaw and Tarek A. M. Nagem," A multi-biometric iris recognition system based on a deep learning approach", Pattern Analysis and Applications, Vol :21,no: 3, pp :783-802, August 2018.

[3] Rathgeb C., Buchmann N., Hofbauer H., Baier H., Uhl A. and Busch C., "Methods for accuracy-preserving acceleration of large-scale comparisons in CPU-based iris recognition systems", IET Biometrics, 2017.

[4] L. Zhang, Q. Li, J. You, D. Zhang, A modified matched filter with double-sided thresholding for screening proliferative diabetic retinopathy, IEEE Transactions on Information Technology in Biomedicine 13 (2009) 528-534

[5] Nadia Othman and Bernadette Dorizzi " Impact of Quality-Based Fusion Techniques for Video-Based Iris Recognition at a Distance", $1556-6013$ (c) 2015 IEEE. Personal use is permitted, but republication/redistribution requires IEEE permission. See http://www.ieee.org/publications_standards/publications/rights/index. $\mathrm{html}$ for more information.

[6] C.N. Doukas, I. Maglogiannis, A.A. Chatziioannou, Computer-supported angiogenesis quantification using image analysis and statistical averaging, IEEE Transactions on Information Technology in Biomedicine 12 (2008)650-657.

[7] K.A. Goatman, A.D. Fleming, S. Philip, G.J. Williams, J.A.Olson, P.F Sharp, Detection of new vessels on the optic discusing retinal photographs, IEEE Transactions on Medical Imaging 30 (2011) 972.

[8] C. Agurto, Y. Honggang, V. Murray, M.S. Pattichis, S. Barriga,W Bauman, et al., Detection of neo vascularization in the optic disc using an AM-FM representation, granulometry, and vessel segmentation, in: Annual International Conference of the IEEE, Engineering in Medicine and Biology Society (EMBC), 2012, pp. 4946-4949.

[9] G. Fahmy, "Super-resolution construction of iris image low resolution face video," in Signal Processing and Its Applications, 2007. ISSPA 2007. 9th International Symposium.

[10] C. Agurto, V. Murray, E. Barriga, S. Murillo, M. Pattichis, H.Davis, et al., Multi scale AM-FM methods for diabetic retinopathy lesion detection, IEEE Transactions on Medical Imaging 29 (2010) 502-512.

[11] Yap, P. T., Raveendran, P., \& Ong, S. H. (2003). Image analysis by Krawtchouk moments. IEEE Transactions on Image Processing, 12(11), 1367-1377.

[12] Sunil Kumar Chawla and Aashish Oberoi, "A Robust Algorithm for Iris Segmentation and Normalization using Hough Transform", In Proceedings of the international Conference on Advanced Computing and Communication Technologies, November 2011.

[13] https://analyticks.wordpress.com/2017/02/07/primer-on-iris-recogniti on/

[14] Chaudhuri, S.: 'Super-resolution imaging' (Kluwer Academic Publishers, 2002)

[15] Park, S., Park, M.K., Kang, M.G., et al.: 'Super-resolution image reconstruction: technical overview', IEEE Signal Process. Mag., 2003, 20, pp. $21-36$

[16] L. Zhang, Q. Li, J. Yu, D. Zhang, A modified matched filter with double-sided thresholding for screening proliferative diabetic retinopathy, IEEE Transactions on Information Technology in Biomedicine 13 (2009) 528-534. 
[17] K.A. Goatman, A.D. Fleming, S. Philip, G.J. Williams, J.A.Olson, P.F. Sharp, Detection of new vessels on the optic disc using retinal photographs, IEEE Transactions on Medical Imaging 30 (2011) 972.

[18] C. Agurto, V. Murray, E. Barriga, S. Murillo, M. Pattichis, H.Davis, et al., Multiscale AM-FM methods for diabetic retinopathy lesion detection, IEEE Transactions on Medical Imaging 29 (2010) 502-512.

[19] C. Agurto, Y. Honggang, V. Murray, M.S. Pattichis, S. Barriga, W. Bauman, et al., Detection of revascularization in the optic disc using an AM-FM representation, granulometry, and vessel segmentation, in: Annual International Conference of the IEEE, Engineering in Medicine and Biology Society (EMBC), 2012, pp. 4946-4949.

[20] K. Nguyen, C. Fookes, S. Sridharan, and S. Denman, "Quality super-resolution for fewer cons trend the move," Information Forensics and Security, IEEE Transactions on vol. 6, no. 4, pp. 1248-1258, Dec 2011.

[21] E. Krichen, S. Garcia-Salicetti, and B. Dorizzi, "A new probabilistic iris quality measure for comprehensive noise detection," Theory, Applications, and Systems, 2007. BTAS 2007. First IEEE International Conference on, Sept 2007, pp. 1

[22] Pillai J.K., Puertas M. and Chellappa R, "Cross-sensor iris recognition through kernel learning", IEEE transactions on pattern analysis and machine intelligence, vol. 36, no. 1, pp.73-85, 2014.

[23] Lim S., Lee K., Byeon O. and Kim T, "Efficient iris recognition through improvement of feature vector and classifier", ETRI journal, vol. 23, no. 2, pp.61-70, 2001.

[24] Zhao Z. and Kumar A., "Towards more accurate iris recognition using deeply learned spatially corresponding features", In Proceedings of the IEEE International Conference on Computer Vision, Venice, Italy, pp. 22-29, October 2017.

[25] Ma L., Wang Y. and Tan T, “ Iris recognition based on multichannel Gabor filtering”, In Proc. Fifth Asian Conf. Computer Vision ,Vol. 1, pp. 279-283, January 2002

[26] B. Jun, I. Choi and D. Kim, "Local Transform Features and Hybridization for Accurate Face and Human Detection," in IEEE Transactions on Pattern Analysis and Machine Intelligence, vol. 35, no. 6, pp. 1423-1436, June 2013.

[27] Jaehan Koh, Venu Govindaraju, and Vipin Chaudhary, "A Robust Iris Localization Method Using an Active Contour Model and Hough Transform", In Proceedings of the 20th International Conference on Pattern Recognition, pp.2852 - 2856, 2010.

[28] Khary Popplewell , Kaushik Roy , Foysal Ahmad , Joseph Shelton, " Multispectral iris recognition utilizing hough transform and modified LBP", In IEEE International Conference on Systems, Man, and Cybernetics (SMC), pp.1396 - 1399, 2014.

[29] Sunil Kumar Chawla and Aashish Oberoi, "A Robust Algorithm for Iris Segmentation and Normalization using Hough Transform", In Proceedings of the international Conference on Advanced Computing and Communication Technologies, November 2011.

[30] Tapabrata Chakraborti, Brendan McCane, Steven Mills and Umapada Pal," LOOP Descriptor: Encoding Repeated Local Patterns for Fine-grained Visual Identification of Lepidoptera", 2017.

[31] Prateekshit Pandey, Richa Singh, and Mayank Vatsa, "Face Recognition using ScatteringWavelet under Illicit Drug Abuse Variations", International Conference on Biometrics (ICB), 25 August 2016.

[32] JensKrommweh, "Tetrolet transform: A new adaptive Haar wavelet algorithm for sparse image representation", Journal of Visual Communication and Image Representation, vol.21, no.4, pp.364-374, May 2010.

[33] Gai-Ge Wang, Suash Deb, Zhihua Cui, "Monarch butterfly optimization", Neural Computing and Applications, pp. 1-20, May 2015.

[34] Labib K. and Vemuri V.R, “An application of principal component analysis to the detection and visualization of computer network attacks", In Annales des telecommunications, vol. 61, no. 1-2, pp. 218-234, Springer-Verlag, February 2006.

[35] Bc. Jan Vojt "Deep neural networks and their implementation", 2016.

[36] CASIA-Iris dataset taken from "http://biometrics.idealtest.org/dbDetailForUser.do?id=4", accessed on August 2018.

[37] A. Alice Nithya and C. Lakshmi, " Enhancing iris recognition framework using feature selection and BPNN", Cluster Computing, pp.1-10.
[38] N. Othman, N. Houmani, and B. Dorizzi, "Improving video recognition via local quality weighted super resolution." Conference on Pattern Recognition Applications and Methods (ICPRAM) SciTePress, 2013, pp. 623.

\section{AUTHORS PROFILE}

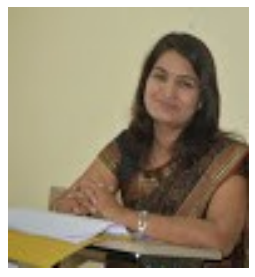

First Author : Ms.Swati D. Shirke holds an M.E.degree in Computer Science and Engineering from Pune University and is a research fellow in the Department of Computer Science and Engineering, Bharath Institute of Higher Education and Research, Bharath University. Her main area of interest includes pattern recognition, image processing, machine learning, She has published several papers in well known peer-reviewed journals.

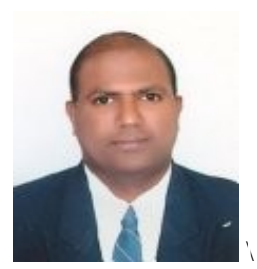

Second Author : Dr. C. Rajabhushnam holds a Ph.D. in Neural Networks, from Louisiana State University, USA And he is a professor in the Department of In the Department of Computer Science and Engineering, Bharath Institute of Higher Education and Research, Bharath University. His main area of interest includes pattern recognition, image processing, and machine learning he has published several papers in well known 